\title{
Planetary Terrestrial Analogues Library (PTAL) project: NIR Characterization of samples
}

\author{
F. Poulet ${ }^{1}$, D. LOIZEAU ${ }^{1}$, C. LANTZ ${ }^{1}$, C. Pilorget ${ }^{1}$, J.- \\ P. BIBRING ${ }^{1}$, V. HAMM ${ }^{1}$ \\ ${ }^{1}$ Université Paris-Saclay, CNRS, Institut d'astrophysique \\ spatiale, 91405, Orsay, France.
}

Multiple spectroscopic techniques have been selected on previous, present and forthcoming missions to explore planetary surfaces in the Solar System. In particular, forthcoming ESA/Roscosmos and NASA missions to the surface of Mars will bring instruments capable of nearinfrared (NIR), Raman and Laser Induced Breakdown Spectroscopies to analyze the mineralogy and chemistry of rocks. The PTAL (Planetary Terrestrial Analogues Library) project aims at building a multi-instrument spectral database of a large variety of natural Earth rock samples, including Mars analogues. Two instruments were selected to characterize the mineralogy of these analogues in the NIR range: a flight spare of NIR hyperspectral microscope MicrOmega/ExoMars and a commercial NIR point spectrometer. The diversity of the PTAL powdered and bulk samples is well revealed by the huge diversity of spectra in the 1-4.0 $\mu \mathrm{m}$. The detected minerals are representative of various formation, alteration and geochemical environments. The major diagnostic features observed are primary silicates like Olivine or Pyroxene with low and/or high Calcium content; various altered silicates detected with vibrational features associated to $\mathrm{OH}$ and/or $\mathrm{H} 2 \mathrm{O}$ and/or metal-OH features; Oxides; Zeolites; Carbonates. In addition, the imaging capability of MicrOmega reveals complex assemblages of these different phases allowing to better constrain the alteration processes at microscopic scale. We will emphasize the multi-analytical study that provides an unique opportunity to coordinate minerals/elements detection by different techniques that are similar to the instruments on board current and forthcoming Martian missions.

The PTAL project is financed through the European Research Council in the H2020-COMPET-2015 program (grant 687302). 